\title{
An Economic User-Centric WiFi Offloading Algorithm for Heterogeneous Network
}

\author{
Jinfeng Kou, Yang Xiao, and Dong Wang \\ Institute of Information Science, Beijing Jiaotong University, Beijing 100044, China \\ Correspondence should be addressed to Jinfeng Kou; xiaokouzi922@126.com
}

Received 10 July 2015; Accepted 21 September 2015

Academic Editor: Yang Tang

Copyright (C) 2015 Jinfeng Kou et al. This is an open access article distributed under the Creative Commons Attribution License, which permits unrestricted use, distribution, and reproduction in any medium, provided the original work is properly cited.

\begin{abstract}
An economic user-centric WiFi offloading algorithm is proposed to satisfy the major concerns of wireless users, who wish to have better network performance with even less network expense. Thus in this paper both system throughput and network expense are considered, and the goal of the proposed offloading algorithm is to obtain an optimal offloading ratio, which can both maximize the system throughput and minimize the network expense. Firstly, a practical system model is set up on the basis of a typical scenario of heterogeneous network. In this model, the average throughput of both cellular network and WiFi network is analyzed carefully. Then an economic user-centric WiFi offloading algorithm is proposed with an evaluation function to evaluate the system, and the optimal offloading ratio can be obtained by minimizing the evaluation function. At last, numerical results represent a direct calculating process of the optimal offloading ratio. These results in return validate the efficiency of the proposed offloading algorithm as well.
\end{abstract}

\section{Introduction}

The explosive growth in mobile data driven by smart devices, such as smart phones and tablets, has stressed conventional cellular network heavily. According to [1], global mobile devices in 2013 have grown to 7 billion, and global wireless data will increase nearly 11-fold between 2013 and 2018, demanding a compound annual growth rate of 61 percent.

To solve this problem, researchers have done a lot and proposed various kinds of solutions, which can be roughly divided into the following three kinds.

(1) Increase Network Coverage via Heterogeneous Network [25]. The main idea of heterogeneous network is to add smallcell networks to complement conventional cellular network, namely, macro/micronetwork. Small-cell networks include picocell network, femtocell network, and Wireless Fidelity (WiFi) [6-11] network. They are normally deployed in dense areas as hot-spots or in edge areas of macro/microbase stations (BSs) as complements.

(2) Increase Subchannel Number via Multi-Input Multioutput (MIMO) Technology [12-16]. MIMO technology uses multiple antennas equipped on the BS to transmit multiple streams of data; in this way channel utilization can be greatly improved and system capacity can be significantly promoted.

(3) Increase Spectrum Utilization via Cognitive Radio (CR) Technology [17-20]. Both licensed cellular spectrum and unlicensed WiFi spectrum have been specified clearly; they are so limited that system performance is greatly restricted. A good solution is to improve spectrum utilization. CR technology encourages secondary users to reuse the unused spectrum of primary users; in this way spectrum can be utilized more efficiently.

In this paper we mainly talk about the first kind solution, namely, using the heterogeneous network. And we use WiFi network as an example of the small-cell networks [10]. The main reason that we select WiFi network instead of other small-cell networks is that WiFi network works in the unlicensed spectrum band of $2.4 \mathrm{GHz}$ and $5 \mathrm{GHz}$; it has no interference to cellular network. While picocell network and femtocell network are working in the same licensed spectrum band with macro/micronetwork, there is 
severe interference between them. Besides, the Long Term Evolution (LTE) [21-23] standard has supported an access network discovery and selection function (ANDSF) [24], which allows user equipment (UE) to sense and get access to WiFi network; thus WiFi network develops dramatically and has been the most widely used small-cell network in current life.

Current WiFi networks can be divided into two categories, namely, the private WiFi network and the public WiFi network. The private WiFi network means privately owned WiFi network; it is always deployed separately in small areas like homes and restaurants. This kind of WiFi network is always deployed in an unorganized way; thus there is severe interference between them as long as their coverages are overlapped. The public WiFi network means network operator owned WiFi network; it is always deployed in dense areas or edges areas as complements of macro/micronetwork. This kind of WiFi network is deployed in an organized way by network operators; thus there is little interference between them. In this paper, we mainly discuss the second kind of WiFi network, namely, the public WiFi network. And unless otherwise specified, we mean the public WiFi network in the following context.

In this paper there is emphasis on the WiFi offloading issue $[10,11]$ of heterogeneous network. In heterogeneous network, users in the overlapped areas can get access to both cellular network and WiFi network; then how to coordinate both networks is a hot topic recently. In this paper, we consider the two major concerns of wireless users, namely, the system throughput and the network expense, and put forward a novel economic user-centric WiFi offloading algorithm for heterogeneous network. In the proposed algorithm, an evaluation function is proposed to evaluate the system. By finding the minimum value of the evaluation function, we can get the optimal WiFi offloading ratio, which can maximize the system throughput with the minimum network expense. In other words, the optimal WiFi offloading ratio can minimize the average network expense per Mbps.

The following context is organized as follows. Related works are reviewed in Section 2. System model for heterogeneous network is proposed in Section 3, in which system throughputs of both cellular network and WiFi network are analyzed carefully. An economic user-centric WiFi offloading algorithm is proposed in Section 4; it is designed to both maximize the system throughput and minimize the network expense. Numerical results and discussions are presented in Section 5 and conclusions are drawn in Section 6.

\section{Related Work}

In heterogeneous network, WiFi offloading is a hot topic recently and has been studied by many researchers [2528]. In [25], a network-assisted user-centric WiFi offloading model was proposed to maximize per-user throughput. In the proposed algorithm, an optimal offloading ratio $\phi^{*}$ is firstly calculated by using network information including user number and packet size. Then users in the overlapped areas transmitted packets through WiFi network with probability $\phi^{*}$ and through cellular network with probability $\left(1-\phi^{*}\right)$. Finally per-user throughput could be maximized. In [26], an energy and content-aware framework was proposed for video transmission in heterogeneous network. The goal of the proposed framework was to guarantee video transmission's quality of service (QoS) under an acceptable energy consumption. The first step of the proposed framework was to allocate the transmission power for each network in consideration of bandwidth, channel condition, and UE's battery constrain. The second step was to do video packet scheduling for each network with a goal of minimizing the video quality distortion.

Both $[25,26]$ provided efficient models for WiFi offloading in heterogeneous network, and they considered many important aspects of wireless network, such as throughput, power consumption, and QoS. However, both of them ignored the economic expense of wireless network, which is a major concern of wireless users. In [27], a distributed WiFi offloading algorithm considering both network utility and network expense was proposed. In the proposed algorithm, network utility was calculated by a logarithmic function of the allocated bandwidth, and network expense was calculated by a linear function of the allocated bandwidth. Utility function was proposed to represent the users' satisfaction; it was a linear function of both network utility and network expense. The goal of the proposed WiFi offloading algorithm was to do bandwidth allocation optimally so that the total satisfaction of all the users could be maximized. In [28], an economic WiFi offloading model was proposed to evaluate the network's benefit of the allocated bandwidth. In the proposed model, network benefit was calculated by a linear function of both network utility and network expense. Network utility was calculated by an exponential function of the needed bandwidth and the allocated bandwidth, and network expense was calculated by a linear function of the allocated bandwidth. The goal of the proposed WiFi offloading algorithm was to allocate bandwidth optimally so that the total network benefit of all the users can be maximized.

Both [27, 28] considered network utility and network expense, but they ignored the fact that network utility and network expense are different concepts; they could not be calculated directly. Thus both utility functions in $[27,28]$ were unreasonable.

In this paper, we put forward a novel economic WiFi offloading algorithm for heterogeneous network. The proposed algorithm considers both system throughput and network expense and uses an evaluation function to evaluate the heterogeneous network. Unlike the above described utility functions, the proposed evaluation function has practical meanings; it represents the average network expense per Mbps. The goal of the proposed WiFi offloading algorithm is to get the minimum value of the evaluation function; in this way system throughput can be maximized and network expense can be minimized. 


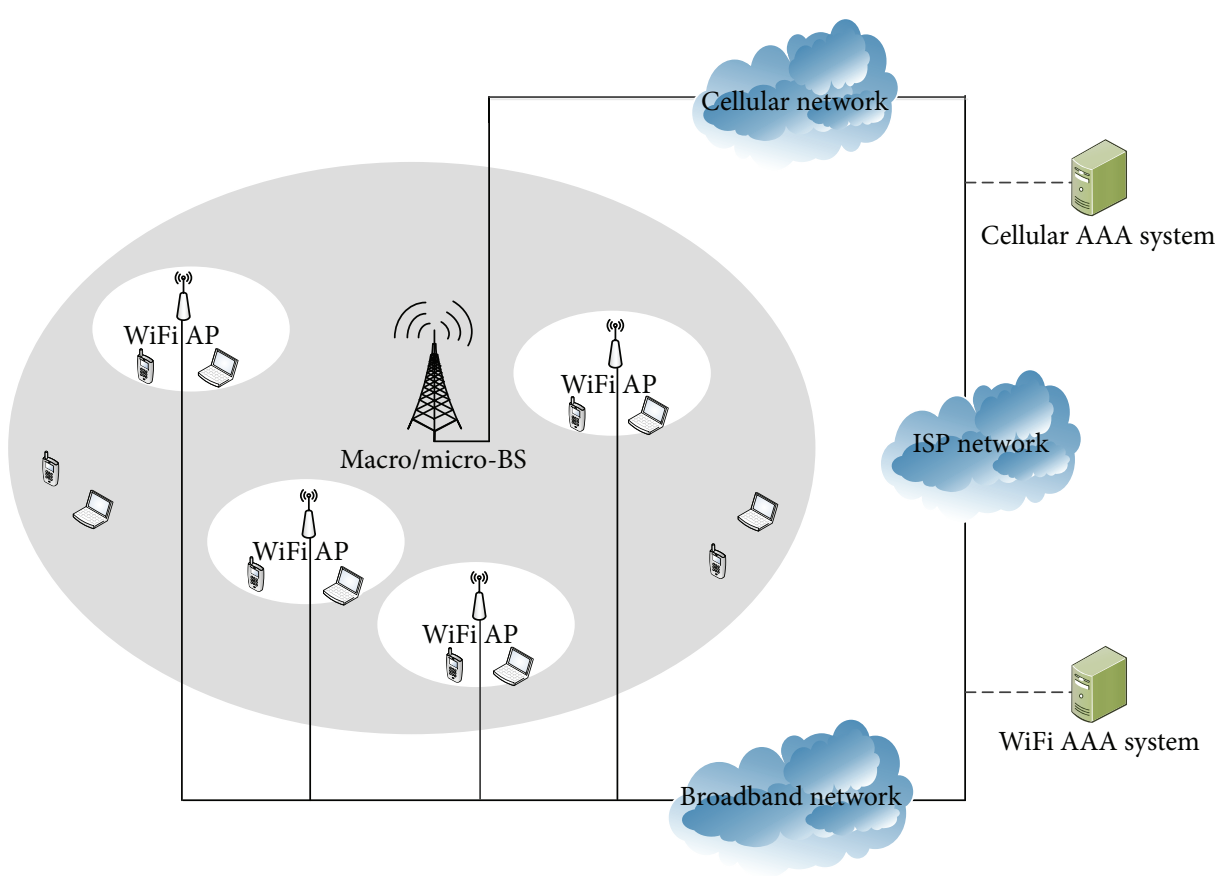

FIGURE 1: A typical scenario of heterogeneous network.

\section{System Model}

Heterogeneous network with both cellular network and WiFi network is a two-layer network, as shown in Figure 1. Cellular network is a basis of heterogeneous network and covers the whole area, while WiFi networks are complements and cover small areas like crowd areas and edge areas. Besides, the two networks are transmitting on different paths. Cellular users get access to internet service provider (ISP) network through cellular network, while WiFi users get access to ISP network through broadband network. They are managed by different authentication, authorization, and accounting (AAA) systems; thus their expense rules are different from each other.

In this paper we mainly talk about heterogeneous networks with one macro/micro-BS and $N_{\mathrm{AP}} \mathrm{WiFi}$ APs. Suppose that the coverage areas of cellular BS and WiFi APs are $S_{C}$ and $S_{W i}$, respectively, $i=1,2, \ldots, N_{\mathrm{AP}}$. As WiFi network discussed in this paper is the public WiFi network, thus WiFi APs are deployed in an organized way and there are no overlapped areas between them; namely, $S_{L} \cap S_{W i}=\emptyset$. Let $S_{\mathrm{CO}}$ be the cellular-only area; then we have

$$
S_{\mathrm{CO}}=S_{C}-\sum_{i=1}^{N_{\mathrm{AP}}} S_{W i}
$$

Suppose that the density of wireless users in cellular network is $\rho_{C}$; the density of wireless users in the $i_{\text {th }} \mathrm{WiFi}$ network is $\rho_{W i}$. The probability of active users in cellular network is $p_{C}$; the probability of active users in the $i_{\text {th }}$ WiFi network is $p_{W i}$. Let $N_{\mathrm{CO}}$ be the number of active users in cellular-only area, and let $N_{C W i}$ be the number of active users in the $i_{\text {th }}$ overlapped area; then we have

$$
\begin{aligned}
N_{\mathrm{CO}} & =\rho_{C} p_{C} S_{\mathrm{CO}}, \\
N_{C W i} & =\rho_{C} p_{C} S_{W i}+\rho_{W i} p_{W i} S_{W i} .
\end{aligned}
$$

Assume that within the user equipment there is a scheduler that collects system parameters and makes the offloading decision. Assume that the WiFi offloading ratio of active users in the overlapped areas is $\lambda, 0 \leq \lambda \leq 1 . \lambda=0$ means that all active users in the overlapped areas are using cellular network, while $\lambda=1$ means that all active users in the overlapped areas are using WiFi network. Let $N_{C}$ be the total number of active users using cellular network, and let $N_{W i}$ be the number of active users using the $i_{\text {th }}$ WiFi network; then

$$
\begin{aligned}
N_{C} & =N_{\mathrm{CO}}+(1-\lambda) N_{\mathrm{AP}} N_{C W i}, \\
N_{W i} & =\lambda N_{C W i} .
\end{aligned}
$$

Let $E\left[\mathrm{Thpt}_{C}\right]$ be the average throughput of active users using cellular network, and let $E\left[\mathrm{Thpt}_{W i}\right]$ be the average throughput of active users using the $i_{\text {th }}$ WiFi network. Then the total throughput of cellular network is

$$
\operatorname{Thpt}_{C}=E\left[\operatorname{Thpt}_{C}\right] N_{C},
$$

the total throughput of WiFi network is

$$
\operatorname{Thpt}_{W}=\sum_{i=1}^{N_{\mathrm{AP}}} E\left[\mathrm{Thpt}_{W i}\right] N_{W i}
$$

and the total throughput of heterogeneous network is

$$
\text { Thpt }=\operatorname{Thpt}_{C}+\operatorname{Thpt}_{W} .
$$


TABLE 1: Subframe configuration (D-downlink, U-uplink, Sspecial).

\begin{tabular}{|c|c|c|}
\hline $\begin{array}{l}\text { Configuration } \\
\text { index }\end{array}$ & $\begin{array}{c}\text { Switch-point } \\
\text { index }\end{array}$ & Subframe index \\
\hline 0 & 5 & $\mathrm{D} S \mathrm{SU} U \mathrm{D} S \mathrm{U} U \mathrm{U}$ \\
\hline 1 & 5 & $\mathrm{D} S \mathrm{~S} U \mathrm{D} \mathrm{D} S \mathrm{~S} U \mathrm{D}$ \\
\hline 2 & 5 & D S U D D D S U D \\
\hline 3 & 10 & D S U U U D D D D D \\
\hline 4 & 10 & D S U U D D D D D D \\
\hline 5 & 10 & D S U D D D D D D D \\
\hline 6 & 5 & $\mathrm{DSU} U \mathrm{U} \mathrm{DS} U \mathrm{U}$ \\
\hline
\end{tabular}

3.1. Average Throughput of Cellular Users. In this paper, we take the latest TD-LTE network as an example of cellular network. In TD-LTE network, medium access control (MAC) layer adopts the scheduled resource allocating mechanism; the average throughput is affected by allocated bandwidth, uplink/downlink subframe configuration, antenna configuration, modulation scheme, and so on [29].

Generally, throughput is defined as the number of efficient data symbols transmitted per second. Thus, in TD-LTE network, the average throughput $E\left[\mathrm{Thpt}_{\mathrm{C}}\right]$ can be expressed as [30]

$$
E\left[\text { Thpt }_{C}\right]=\frac{N_{\text {stream }}^{C} M_{\text {mod }}^{C} N_{E B}}{T_{\text {frame }}},
$$

where $N_{\text {stream }}^{C}$ is the stream number that is decided by antenna configuration. $M_{\text {mod }}^{C}$ is the modulation order. $N_{E B}$ is the number of efficient data symbols (regardless of control signals) transmitted per frame. $T_{\text {frame }}$ is the duration of a frame.

As is defined in [29], in TD-LTE network each data frame consists of 10 subframes and each subframe consists of 2 slots. Subframes can be divided into three categories: uplink subframe for uplink transmission, downlink subframe for downlink transmission, and special subframe for system control. Table 1 shows the whole subframe configurations.

In each slot, the transmitted signals can be described by a resource grid of $N_{\mathrm{SC}}$ subcarriers and $N_{S}$ symbols, where $N_{\text {SC }}$ is the allocated subcarrier number per user and $N_{S}$ is the symbol number transmitted per subcarrier per slot, as shown in Figure 2. Thus the number of efficient data symbols transmitted per frame can be expressed as

$$
N_{E B}=N_{\text {SC }} N_{S} \text {, }
$$

where $N_{\mathrm{SC}}$ depends on the system's subcarrier number and user number. Table 2 shows the system's subcarrier numbers under different system bandwidth conditions.

3.2. Average Throughput of WiFi Users. Bianchi's Markov model [31] is considered the most efficient model for analyzing the distributed coordination function (DCF) process in 802.11 network, but it still has significant shortages:

(1) As is defined in [8], there is a retransmission limit in the retransmission process. If the packet has not
TABLE 2: System's subcarrier number under different system bandwidth conditions.

\begin{tabular}{lcccccc}
\hline System bandwidth $(\mathrm{MHz})$ & 1.4 & 3 & 5 & 10 & 15 & 20 \\
\hline System's RB number & 6 & 15 & 25 & 50 & 75 & 100 \\
\hline System's subcarrier number & 72 & 180 & 300 & 600 & 900 & 1200
\end{tabular}

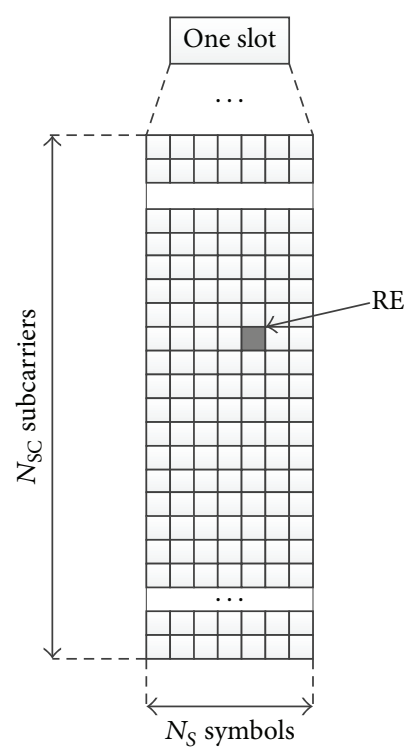

FIgURE 2: Resource grid in TD-LTE network.

be transmitted within the retransmission limit, it will be discarded. But in Bianchi's Markov model, the retransmission limit is not considered; packets could be retransmitted forever with the maximum contention window $C W_{\max }$.

(2) As is defined in [8], there is a frozen back-off scheme in the back-off process. If the channel has been idle for a DIFS, the back-off counter reduces with 1 . Otherwise, if the channel is sensed busy during a DIFS, the back-off counter stops reducing and keeps current value. This value will be reduced again only when the channel is sensed idle for a DIFS. But in Bianchi's Markov model, the frozen back-off scheme is not considered; the back-off counter is always uniformly chosen in the range of $(0, \omega-1)$, where $\omega$ is the size of the contention window.

Considering these two aspects, we decide to improve Bianchi's Markov model with the following model described in Figure 3. In the improved Markov model, $p$ is the probability that a packet meets a collision on the channel. $W_{0}$ is the minimum size of the contention window, $W_{0}=C W_{\min }$. $W_{m^{\prime}}$ is the maximum size of the contention window, $W_{m^{\prime}}=$ $C W_{\max }=2^{m^{\prime}} W_{0} \cdot m$ is the retransmission limit, $W_{m}=2^{m} W_{0}$. 


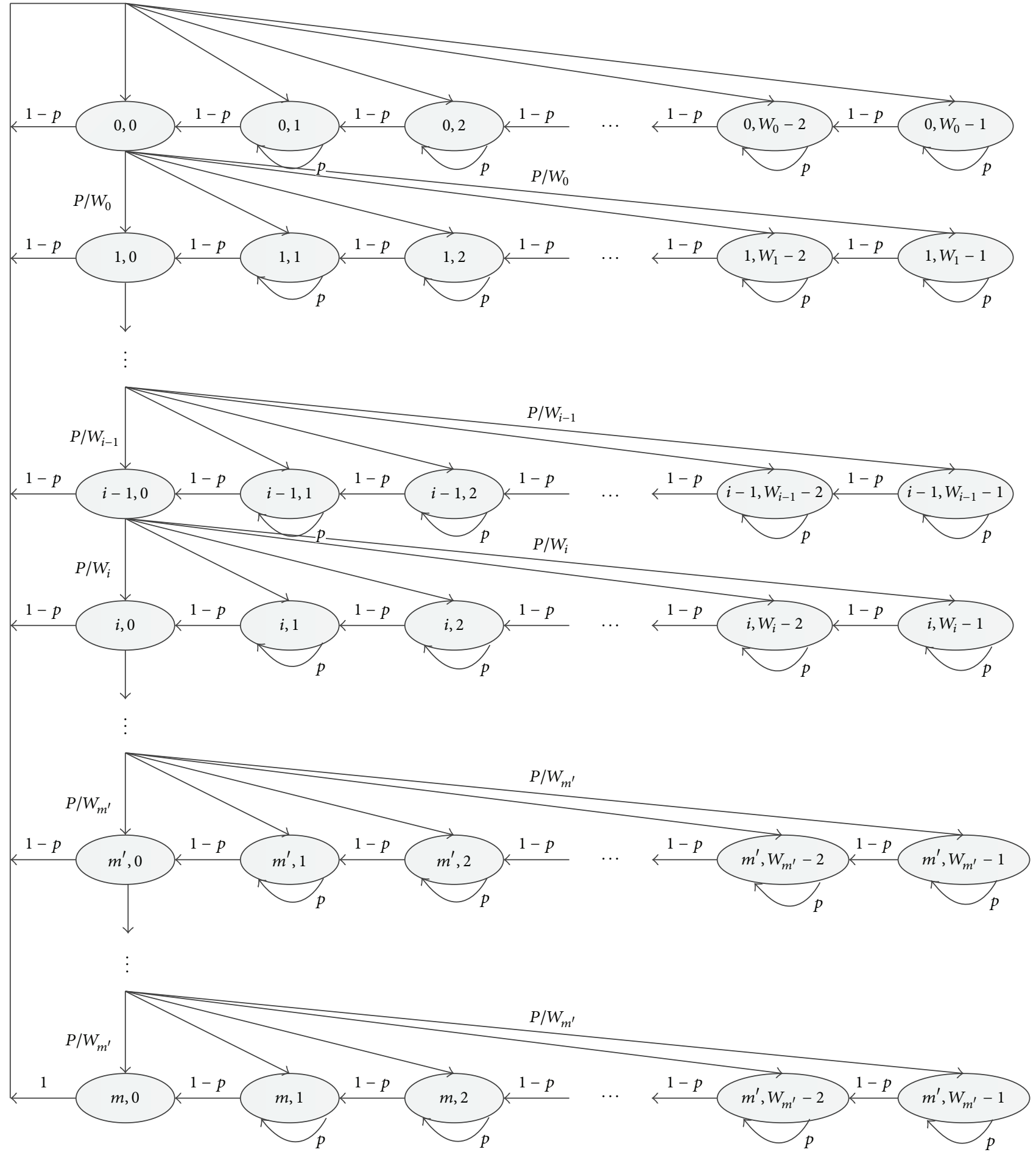

FIgUre 3: The improved Markov model. 
$W_{i}$ is the size of the contention window in the $i_{\text {th }}$ back-off stage; it satisfies

$$
\begin{aligned}
& W_{i}=2^{i} W_{0}, \quad i \leq m^{\prime} \\
& W_{i}=W_{m^{\prime}}=2^{m^{\prime}} W_{0}, \quad i>m^{\prime} .
\end{aligned}
$$

Equation (9) means that once the contention window increases to the maximum value $W_{m^{\prime}}$, it will stop increasing and keep this value in the following retransmission process.

In this improved Markov model, the one-step transition processes are

$$
\begin{aligned}
P\{i, k \mid i, k+1\} & =1-p, \\
P\{i, k \mid i, k\} & =p, \quad i \in[0, m], k \in\left[0, W_{m}-1\right), \\
P\{i, k \mid i-1,0\} & =\frac{p}{W_{i}}, \quad i \in(0, m], k \in\left[0, W_{m}-1\right], \\
P\{0, k \mid i, 0\} & =\frac{1-p}{W_{0}}, \\
P\{0, k \mid m, k\} & =\frac{1}{W_{0}}, \quad i \in[0, m], k \in\left[0, W_{0}-1\right] .
\end{aligned}
$$

The first equation in (10) means that the channel is sensed idle and the back-off counter decreases. The second equation means that the back-off counter stops reducing because the channel is sensed busy. The third equation means that an unsuccessful transmission occurs in the $(i-1)_{\text {th }}$ back-off stage and the back-off stage increases. The forth equation means that the station starts a new back-off process at the end of a successful transmission. The fifth equation means that the station starts a new back-off process because of the retransmission limit.

Let $b_{i, k}=\lim _{t \rightarrow \infty} P\{i, k\}, i \in[0, m], k \in\left[0, W_{i}-1\right]$, be the stationary distribution of the Markov chain. Then we have

$$
b_{i-1,0} p=b_{i, 0}, \quad i \in(0, m) \text {; }
$$

by using the recursive method, we can get

$$
b_{i, 0}=p^{i} b_{0,0}, \quad i \in[0, m] ;
$$

besides, for each $k \in\left(1, W_{i}-1\right], b_{i, k}$ satisfies

$$
\begin{aligned}
& (1-p) b_{0, k}=\frac{W_{0}-k}{W_{0}}\left[(1-p) \sum_{j=0}^{m-1} b_{j, 0}+b_{m, 0}\right], \\
& (1-p) b_{i, k}=\frac{W_{i}-k}{W_{i}} b_{i-1,0} p, \quad i>0
\end{aligned}
$$

with (12), (13) can be rewritten as

$$
b_{i, k}=\frac{W_{i}-k}{W_{i}(1-p)} b_{i, 0}, \quad i \in[0, m], k \in\left(0, W_{i}\right] .
$$

As the total probability of all states in Markov chain equals 1, we have

$$
1=\sum_{i=0}^{m} \sum_{k=0}^{W_{i}-1} b_{i, k}=\sum_{i=0}^{m}\left[p^{i} b_{i, 0}+\sum_{k=1}^{W_{i}-1} \frac{W_{i}-k}{W_{i}(1-p)} b_{i, k}\right] ;
$$

with (9), (12), and (14), we can get (16)

$$
\begin{aligned}
& b_{0,0}=\frac{2(1-p)^{2}(1-2 p)}{c c}, \quad m \geq m^{\prime}, \\
& b_{0,0}=\frac{2(1-p)^{2}(1-2 p)}{c c+2^{m^{\prime}} W_{0}(1-2 p)\left(p^{m^{\prime}+1}-p^{m+1}\right)},
\end{aligned}
$$

$m<m^{\prime}$

where $c c=\left(1-p^{m+1}\right)(1-2 p)^{2}+W_{0}(1-p)\left(1-(2 p)^{m+1}\right)$; let $\tau$ be the probability that the station can access the channel in an arbitrary slot time; then $\tau$ satisfies

$$
\tau=\sum_{i=0}^{m} b_{i, 0}=\frac{b_{0,0}}{1-p}
$$

note that $p$ is the probability that a packet meets a collision on the channel; that is to say, there is at least one station transmitting packets in the same slot time. Namely, $p$ satisfies

$$
p=1-(1-\tau)^{n-1}
$$

where $n$ is the user number in this WiFi AP. Equations (17) and (18) form a nonliner equation group with two unknown variables $\tau$ and $p$; thus $\tau$ and $p$ can be calculated by solving (17) and (18).

According to [31], the average throughput of 802.11 network can be expressed as

$$
E\left[\operatorname{Thpt}_{W i}\right]=\frac{P_{s} P_{\mathrm{tr}} E[P]}{\left(1-P_{\mathrm{tr}}\right) \sigma+P_{\mathrm{tr}} P_{s} T_{s}+P_{\mathrm{tr}}\left(1-P_{s}\right) T_{c}},
$$

where $P_{\mathrm{tr}}$ is the probability that there is at least one transmission in an arbitrary slot time. $P_{s}$ is the probability that there is a successful transmission on the channel. Thus $P_{\text {tr }}$ and $P_{s}$ satisfy

$$
\begin{aligned}
& P_{\mathrm{tr}}=1-(1-\tau)^{n}, \\
& P_{s}=\frac{n \tau(1-\tau)^{n-1}}{P_{\mathrm{tr}}}=\frac{n \tau(1-\tau)^{n-1}}{1-(1-\tau)^{n}} .
\end{aligned}
$$

$E[P]$ is the payload size of the transmitting packet. $\sigma$ is the duration of an empty slot. $T_{s}$ is the time consumed by a successful transmission. $T_{c}$ is the time consumed by a collision.

In the basic mechanism, $T_{s}$ and $T_{c}$ satisfy

$$
\begin{aligned}
& T_{s}=T_{\text {header }}+\frac{E[P]}{R_{W}}+T_{\mathrm{SIFS}}+\delta+T_{\mathrm{ACK}}+T_{\mathrm{DIFS}}+\delta, \\
& T_{c}=T_{\text {header }}+\frac{E\left[P^{*}\right]}{R_{W}}+T_{\mathrm{DIFS}}+\delta,
\end{aligned}
$$


where $T_{\text {header }}$ is the time consumed by MAC header and physical layer (PHY) header. $R_{W}$ is the channel rate of 802.11 network. $T_{\text {SIFS }}, T_{\text {ACK }}$, and $T_{\text {DIFS }}$ are the time consumed by SIFS, an ACK frame, and a DIFS, respectively. $\delta$ is the propagation delay, and $E\left[P^{*}\right]$ is the size of the longest payload involved in a collision.

In the RTS/CTS mechanism, $T_{s}$ and $T_{c}$ satisfy

$$
\begin{aligned}
T_{s}= & T_{\mathrm{RTS}}+T_{\mathrm{SIFS}}+\delta+T_{\mathrm{CTS}}+T_{\mathrm{SIFS}}+\delta+T_{\text {header }} \\
& +\frac{E[P]}{R_{W}}+T_{\mathrm{SIFS}}+\delta+T_{\mathrm{ACK}}+T_{\mathrm{DIFS}}+\delta, \\
T_{c}= & T_{\mathrm{RTS}}+T_{\mathrm{DIFS}}+\delta
\end{aligned}
$$

where $T_{\text {RTS }}$ and $T_{\text {CTS }}$ are the time consumed by a RTS frame and a CTS frame, respectively.

However, Bianchi's conclusion on WiFi throughput is not comprehensive because it ignores the effects of antenna configuration and modulation process. Thus in this paper we modify (19) with the following equation:

$$
E\left[\operatorname{Thpt}_{W i}\right]=\frac{P_{s} P_{\mathrm{tr}} E[P] N_{\text {stream }}^{W} M_{\mathrm{mod}}^{W}}{\left(1-P_{\mathrm{tr}}\right) \sigma+P_{\mathrm{tr}} P_{s} T_{s}+P_{\mathrm{tr}}\left(1-P_{s}\right) T_{c}},
$$

where $N_{\text {stream }}^{W}$ is the stream number of WiFi networks and $M_{\text {mod }}^{W}$ is the modulation order of WiFi network.

\section{The Economic User-Centric WiFi Offloading Algorithm}

In this section, the problem of WiFi offloading in heterogeneous network is discussed. It is well known that wireless users intend to have much better performance with even less expense; thus in this paper we evaluate the heterogeneous network with both aspects of system throughput and network expense. A novel user-centric economic WiFi offloading algorithm is described in this section; it can maximize the throughput of heterogeneous network with the minimum expense.

Let $f(\lambda)$ be the evaluation function of the heterogeneous network with the offloading ratio being $\lambda$. Considering both aspects of system throughput and network expense, we define $f(\lambda)$ as

$$
f(\lambda)=\frac{h(\lambda)}{\operatorname{Thpt}(\lambda)}=\frac{h_{C}(\lambda)+h_{W}(\lambda)}{\operatorname{Thpt}_{C}(\lambda)+\operatorname{Thpt}_{W}(\lambda)},
$$

where $h(\lambda)$ represents the total expense consumed by heterogeneous network; it includes the expense consumed by cellular network $h_{C}(\lambda)$ and the expense consumed by WiFi network $h_{W}(\lambda)$. The goal of the proposed offloading algorithm is to get both the maximum value of $\operatorname{Thpt}(\lambda)$ and the minimum value of $h(\lambda)$, namely, to get the minimum value of $f(\lambda)$.

As $\operatorname{Thpt}(\lambda)$ can be calculated by (6), thus $h(\lambda)$ should be determined firstly in order to get the exact expression of $f(\lambda)$. In fact, network expense has a relationship with network throughput. Table 3 shows an example of this relationship, which is exactly used in China Mobile for cellular network.
TABLE 3: Expense for cellular network in China Mobile.

\begin{tabular}{llllllllll}
\hline $\begin{array}{l}\text { System } \\
\text { throughput } \\
\text { (MHz) }\end{array}$ & 10 & 30 & 70 & 150 & 500 & 700 & 1000 & 2000 & 3000 \\
\hline $\begin{array}{l}\text { Network } \\
\text { expense (Yuan) }\end{array}$ & 3 & 5 & 10 & 20 & 30 & 40 & 50 & 70 & 100 \\
\hline
\end{tabular}

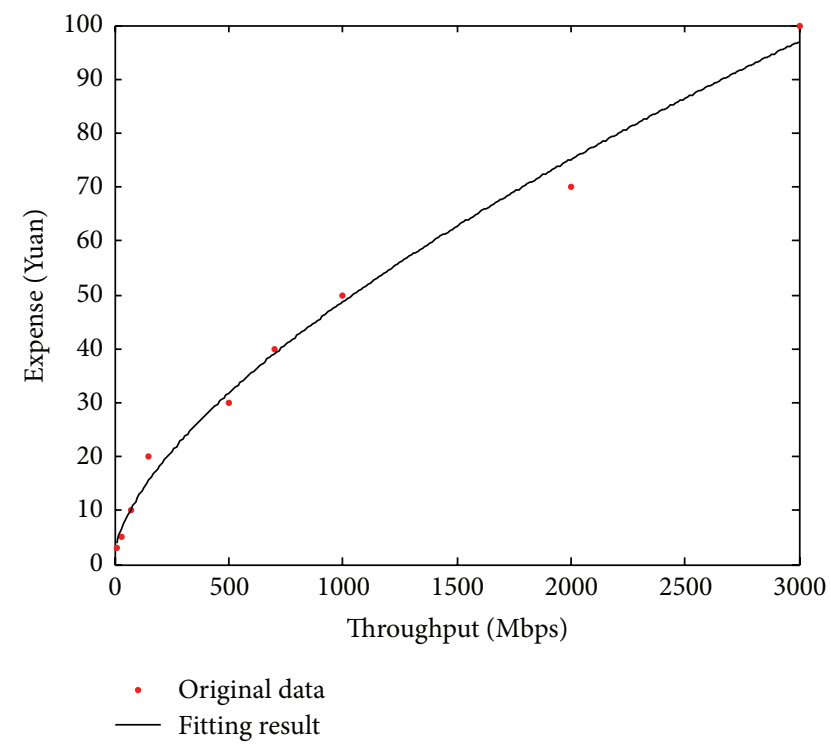

FIgURE 4: Fitting results versus original data.

Using the data in Table 3, we can fit the relationship between network expense and system throughput. In this paper, we use the power series in (25) to fit the relationship. One has

$$
h_{C}(\lambda)=a_{C} \operatorname{Thpt}_{C}(\lambda)^{b_{C}}+c_{C}
$$

Fitting result is shown in Figure 4 , where $a_{C}, b_{C}$, and $c_{C}$ are $0.5559,0.6426$, and 1.618 , respectively. Figure 4 shows that the power fitting result matches the original data perfectly; thus the power fitting method is proved to be efficient and it can be used in the following fitting process of WiFi network.

Similarly, the relationship between WiFi network expense and WiFi throughput can be fitted as

$$
h_{W}(\lambda)=a_{W} \operatorname{Thpt}_{W}(\lambda)^{b_{W}}+c_{W}
$$

with (24)-(26), it is easy to conclude that $F(\lambda)$ is a function of only one variable, offloading ratio $\lambda$; thus the optimal $\lambda^{*}$ can be calculated by solving $F\left(\lambda^{*}\right)=\min [f(\lambda)]$. In this paper we prefer to use the enumeration method to get $\lambda^{*}$, because the enumeration method is more direct and can be understand more easily. Section 5 represents our calculating process.

\section{Numerical Results and Discussions}

In this section, we represent a direct calculating process for the proposed economic user-centric WiFi offloading 
TABLE 4: System parameters.

\begin{tabular}{|c|c|c|c|}
\hline $\begin{array}{l}\text { Cellular } \\
\text { parameters }\end{array}$ & Values & WiFi parameters & Values \\
\hline $\begin{array}{l}\text { Bandwidth } \\
(\mathrm{MHz})\end{array}$ & 20 & Bandwidth (MHz) & 20 \\
\hline BS number & 1 & $N_{\mathrm{AP}}$ & 4 \\
\hline$S_{C}\left(\mathrm{~m}^{2}\right)$ & $1000 \times 1000$ & $S_{W i}\left(\mathrm{~m}^{2}\right)$ & $100 \times 100$ \\
\hline$\rho_{C}\left(\mathrm{~m}^{2}\right)$ & $5 \times 10^{-4}$ & $\rho_{W i}\left(\mathrm{~m}^{2}\right)$ & $5 \times 10^{-3}$ \\
\hline$p_{C}$ & 0.1 & $p_{W i}$ & 0.2 \\
\hline$T_{\text {frame }}(\mathrm{ms})$ & 10 & $E[P]$ (bite) & 1024 \\
\hline$N_{\text {stream }}^{C}$ & 2 & $N_{\text {stream }}^{W}$ & 2 \\
\hline$M_{\mathrm{mod}}^{\mathrm{C}}$ & 6 (64QAM) & $M_{\mathrm{mod}}^{W}$ & 4 (16QAM) \\
\hline Subframe & Configuration 1 & $R_{W}(\mathrm{Mbps})$ & $108(802.11 n)$ \\
\hline \multirow[t]{5}{*}{$N_{S}$} & 14 & $W_{0}, W_{m^{\prime}}, m$ & $16,2048,10$ \\
\hline & & $T_{\text {header }}, T_{\mathrm{ACK}}$ (us) & 464,304 \\
\hline & & $T_{\mathrm{RTS}}, T_{\mathrm{CTS}}(\mathrm{us})$ & 352,304 \\
\hline & & $T_{\mathrm{DIFS}}, T_{\mathrm{SIFS}}$ (us) & 50,10 \\
\hline & & $\sigma, \delta$ (us) & 20,1 \\
\hline
\end{tabular}

TABle 5: Expense for WiFi network in China Mobile.

\begin{tabular}{lcccccc}
\hline $\begin{array}{l}\text { System } \\
\text { throughput } \\
\text { (MHz) }\end{array}$ & 300 & 700 & 1500 & 2800 & 5000 & 20000 \\
\hline $\begin{array}{l}\text { Network } \\
\text { expense (Yuan) }\end{array}$ & 5 & 10 & 20 & 30 & 50 & 100 \\
\hline
\end{tabular}

algorithm. Numerical results show the effects of offloading ratio on system throughput and network expense, and the optimal offloading ratio $\lambda^{*}$ can be obtained from these results directly.

A heterogeneous network with one cellular BS and $4 \mathrm{WiFi}$ APs is considered in this experiment. And for simplicity, we assume that parameters of different WiFi APs are of the same value. Namely, the coverage areas $S_{W i}$ are of the same value, the density of wireless users $\rho_{W i}$ is of the same value, and the probability of active users $p_{W i}$ is of the same value, $i=$ $1,2, \ldots, N_{\mathrm{AP}}$. Table 4 shows the parameters in detail.

We use the above expense data in Table 3 for cellular network and the following expense data in Table 5 for WiFi network $\left(a_{W}, b_{W}\right.$, and $c_{W}$ are 1.07, 0.4703, and -12.28 , resp.); both of these data are from China Mobile. Then we calculate the system throughput and network expense respectively; calculating results are shown in Figures 5-7. Note that, for simplicity, in this experiment we use 0.1 as an interval of the offloading ratio, so the accuracy of the offloading ratio in this experiment is 0.1 . But this interval can be set smaller to meet the more accurate requirements.

Figure 5 shows the throughput results of cellular network, WiFi network, and heterogeneous network. As cellular network adopts the scheduled resource allocation mechanism, all bandwidth is utilized perfectly no matter how many users are offloaded to cellular network; thus the total throughput

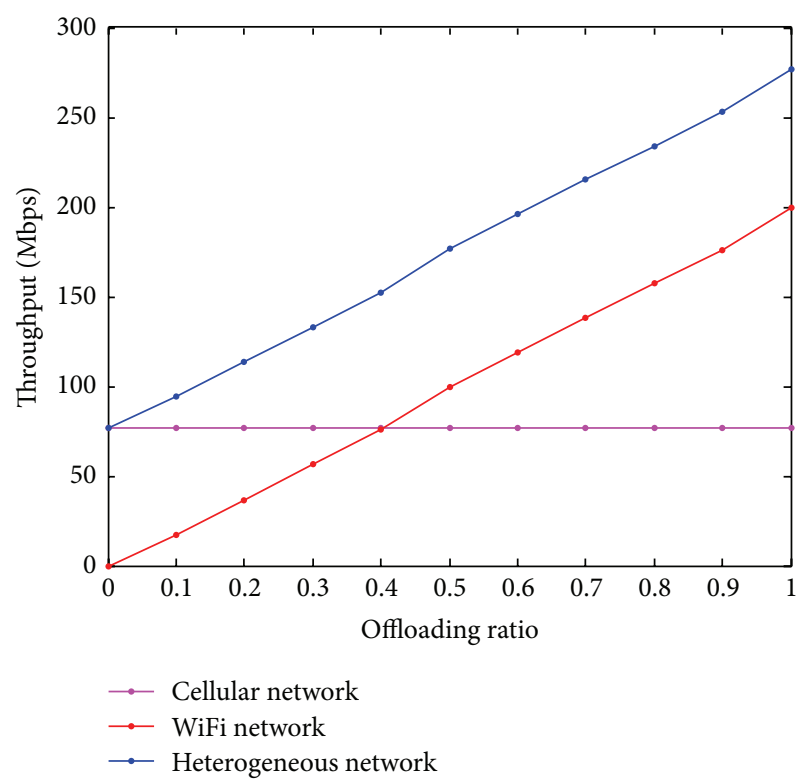

FIGURE 5: Throughput results.

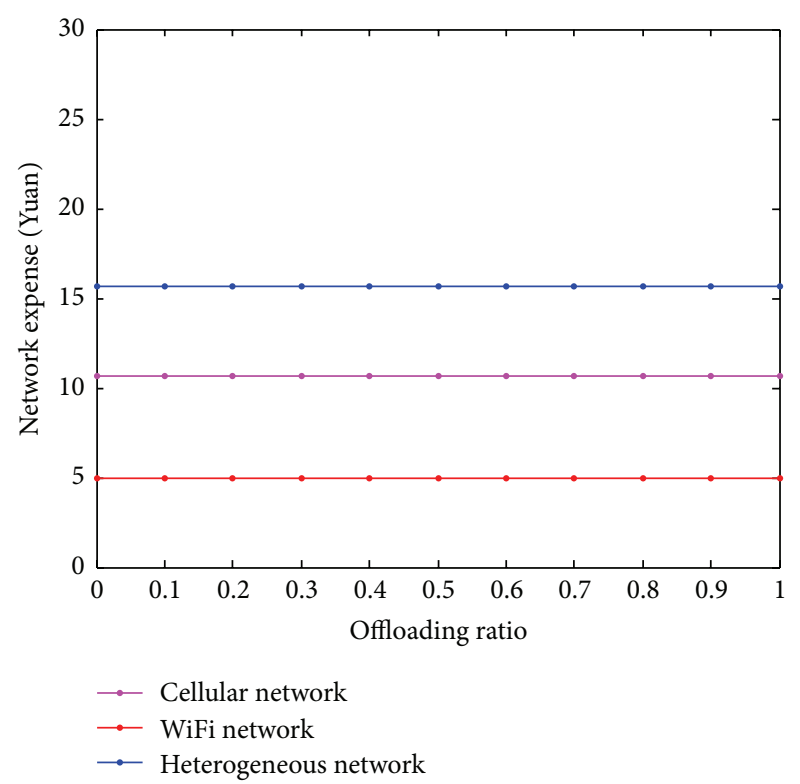

FIGURE 6: Network expense results.

of cellular network remains the same value during the whole offloading process, while, for WiFi network, it is far from being saturated (10 users per AP); thus its throughput increases linearly with the user number, namely, the offloading ratio. As a result, the whole throughput of heterogeneous network increases linearly with the offloading ratio too.

Figure 6 shows the network expense results of cellular network, WiFi network, and heterogeneous network. As the throughput of cellular network remains the same value during the whole offloading process, its network expense keeps the same value as well, while, for WiFi network, its throughput is less than $300 \mathrm{Mbps}$ during the whole offloading 


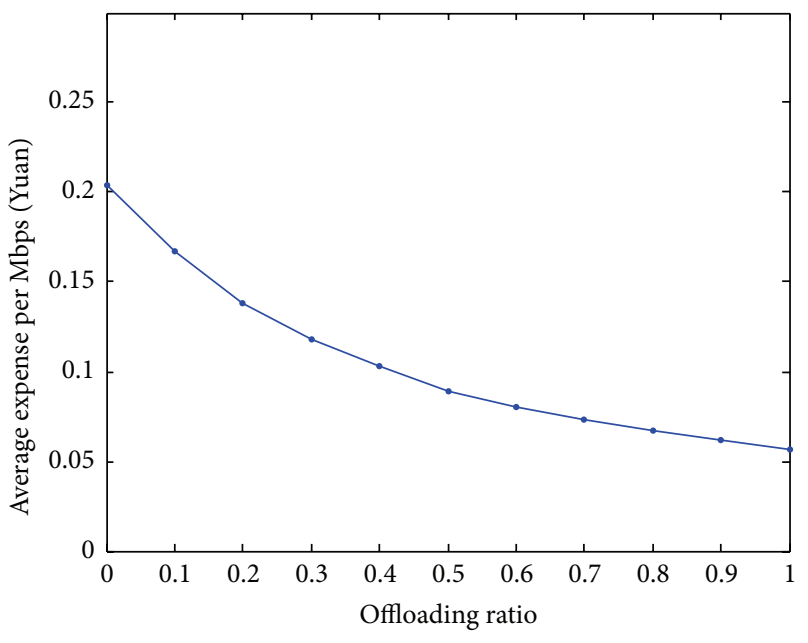

Figure 7: Average expense per Mbps.

process; thus its expense keeps at 5 Yuan. As a result, the total expense of heterogeneous network keeps at about 16 Yuan.

Figure 7 shows the average expenses per Mbps with different offloading ratios. As shown in Figures 5 and 6, the total throughput of heterogeneous network increases with the increasing of offloading ratio and the total expense of heterogeneous network remains the same; thus the average expense per Mbps decreases with the increasing of offloading ratio.

According to Figure 7, it is easy to conclude that the optimal offloading ratio in this experiment is 1 , because the average expenses per Mbps with other offloading ratios are much higher. In return, these results in Figures 5-7 also prove that the proposed WiFi offloading algorithm could both maximize the system throughput and minimize the network expense. Thus the proposed WiFi offloading algorithm is proved to be efficient.

Note that the optimal offloading ratio $\lambda^{*}=1$ is obtained with the above described system parameters; it may be different from other scenarios which adopts different expense rules and different system parameters. But this calculating process is general and can be used in other scenarios.

\section{Conclusion}

In this paper we make a research on the WiFi offloading issue of heterogeneous network. By considering both aspects of system throughput and network expense, we put forward a novel user-centric economic WiFi offloading algorithm. In the proposed algorithm, an evaluation function is used to evaluate the system, and the optimal offloading ratio can be calculated by finding the minimum value of the evaluation function. Numerical results represents a direct calculating process of the optimal offloading ratio, and in turn they validate the efficiency of the proposed WiFi offloading algorithm.

Note that the proposed offloading algorithm is performed under the assumption that there is a scheduler equipped within the user equipment; thus an additional component should be added to user equipment to perform WiFi offloading process. This may bring additional stress on user equipment.

For simplicity, our system modeling, analysis, and simulation are all focusing on the single-BS scenario. In the future we will go a step further and make research on the multi-BSs scenarios. In multi-BSs scenarios more system parameters, such as moving speed and handoff process, should be taken into consideration too.

\section{Conflict of Interests}

The authors declare that there is no conflict of interests regarding the publication of this paper.

\section{Acknowledgments}

This paper is supported by the Fundamental Research Funds for the Central Universities no. 2014JBM023, the National Natural Science Foundation of China no. 61106022, and Beijing Natural Science Foundation no. 4143066.

\section{References}

[1] Cisco, Cisco Visual Networking Index: Global Mobile Data Traffic Forecast Update, 2013-2018, Cisco, San Francisco, Calif, USA, 2014.

[2] A. Ghosh, N. Mangalvedhe, R. Ratasuk et al., "Heterogeneous cellular networks: from theory to practice," IEEE Communications Magazine, vol. 50, no. 6, pp. 54-64, 2012.

[3] V. Suryaprakash, J. Møller, and G. Fettweis, "On the modeling and analysis of heterogeneous radio access networks using a Poisson cluster process," IEEE Transactions on Wireless Communications, vol. 14, no. 2, pp. 1035-1047, 2015.

[4] M. Peng, Y. Li, J. Jiang, J. Li, and C. Wang, "Heterogeneous cloud radio access networks: a new perspective for enhancing spectral and energy efficiencies," IEEE Wireless Communications, vol. 21, no. 6, pp. 126-135, 2014.

[5] A. Kumar, R. K. Mallik, and R. Schober, "A probabilistic approach to modeling users' network selection in the presence of heterogeneous wireless networks," IEEE Transactions on Vehicular Technology, vol. 63, no. 7, pp. 3331-3341, 2014.

[6] IEEE 802.11, “Wireless LAN medium access control (MAC) and physical layer (PHY) specifications: high-speed physical layer extension in the $5 \mathrm{GHz}$ band," 1999.

[7] IEEE, "Wireless LAN medium access control (MAC) and physical layer (PHY) specifications: high-speed physical layer extension in the $2.4 \mathrm{GHz}$ band," IEEE 802.11a, IEEE-SA Standards Board, 2000.

[8] IEEE 802.11b, "Wireless LAN medium access control (MAC) and physical layer (PHY) specifications: high-speed physical layer extension in the $2.4 \mathrm{GHz}$ band," 2000.

[9] IEEE 802.11n, "Wireless LAN medium access control (MAC) and physical layer (PHY) specifications: enhancements for higher throughput," 2009.

[10] M. Bennis, M. Simsek, A. Czylwik, W. Saad, S. Valentin, and M. Debbah, "When cellular meets WiFi in wireless small cell networks," IEEE Communications Magazine, vol. 51, no. 6, pp. 44-50, 2013. 
[11] O. Galinina, A. Pyattaev, S. Andreev, M. Dohler, and Y. Koucheryavy, "5G multi-RAT LTE-WiFi ultra-dense small cells: performance dynamics, architecture, and trends," IEEE Journal on Selected Areas in Communications, vol. 33, no. 6, pp. 12241240, 2015.

[12] S. A. Ramprashad, H. C. Papadopoulos, A. Benjebbour, Y. Kishiyama, N. Jindal, and G. Caire, "Cooperative cellular networks using multi-user MIMO: trade-offs, overheads, and interference control across architectures," IEEE Communications Magazine, vol. 49, no. 5, pp. 70-77, 2011.

[13] C. Lim, T. Yoo, B. Clerckx, B. Lee, and B. Shim, "Recent trend of multiuser MIMO in LTE-advanced," IEEE Communications Magazine, vol. 51, no. 3, pp. 127-135, 2013.

[14] L. Liu, R. Chen, S. Geirhofer, K. Sayana, Z. Shi, and Y. Zhou, "Downlink MIMO in LTE-advanced: SU-MIMO vs. MUMIMO," IEEE Communications Magazine, vol. 50, no. 2, pp. 140-147, 2012.

[15] T. L. Marzetta, "Noncooperative cellular wireless with unlimited numbers of base station antennas," IEEE Transactions on Wireless Communications, vol. 9, no. 11, pp. 3590-3600, 2010.

[16] E. G. Larsson, O. Edfors, F. Tufvesson, and T. L. Marzetta, "Massive MIMO for next generation wireless systems," IEEE Communications Magazine, vol. 52, no. 2, pp. 186-195, 2014.

[17] S. Akin and M. C. Gursoy, "Performance analysis of cognitive radio systems with imperfect channel sensing and estimation," IEEE Transactions on Communications, vol. 63, no. 5, pp. 15541566, 2015.

[18] J. Lunden, M. Motani, and H. V. Poor, "Distributed algorithms for sharing spectrum sensing information in cognitive radio networks," IEEE Transactions on Wireless Communications, vol. 14, no. 8, pp. 4667-4678, 2015.

[19] R. Combes and A. Proutiere, "Dynamic rate and channel selection in cognitive radio systems," IEEE Journal on Selected Areas in Communications, vol. 33, no. 5, pp. 910-921, 2015.

[20] X. Hong, J. Wang, C.-X. Wang, and J. Shi, "Cognitive radio in 5G: a perspective on energy-spectral efficiency trade-off," IEEE Communications Magazine, vol. 52, no. 7, pp. 46-53, 2014.

[21] 3GPP TS 36.300, "Evolved Universal Terrestrial Radio Access (E-UTRA) and Evolved Universal Terrestrial Radio Access Network (E-UTRA); Overall description (Release 10)," 2010.

[22] 3GPP TS36.321, "Evolved universal terrestrial radio access (EUTRA); Medium access control (MAC) protocol specification”.

[23] 3GPP TR36.201, "Evolved universal terrestrial radio access (EUTRA); LTE physical layer; General description (Release 9)," 2010.

[24] 3GPP TS24.312, "Access network discovery and selection function (ANDSF) management object (Release 11)," 2012.

[25] B. H. Jung, N.-O. Song, and D. K. Sung, "A network-assisted user-centric WiFi-offloading model for maximizing per-user throughput in a heterogeneous network," IEEE Transactions on Vehicular Technology, vol. 63, no. 4, pp. 1940-1945, 2014.

[26] M. Ismail, W. Zhuang, and S. Elhedhli, "Energy and content aware multi-homing video transmission in heterogeneous networks," IEEE Transactions on Wireless Communications, vol. 12, no. 7, pp. 3600-3610, 2013.

[27] M. Ismail and W. Zhuang, "A distributed multi-service resource allocation algorithm in heterogeneous wireless access medium," IEEE Journal on Selected Areas in Communications, vol. 30, no. 2, pp. 425-432, 2012.

[28] X. Pei, T. Jiang, D. Qu, G. Zhu, and J. Liu, "Radio-resource management and access-control mechanism based on a novel economic model in heterogeneous wireless networks," IEEE Transactions on Vehicular Technology, vol. 59, no. 6, pp. 30473055, 2010.

[29] 3GPP, "Evolved universal terrestrial radio access (E-UTRA): physical channels and modulation (release 10)," 3GPP TS 36.211, 3GPP, 2011.

[30] China International Telecommunication Construction Group Design Institute, LTE Networking and Engineering Practice, Post and Telecom Press, Beijing, China, 2014.

[31] G. Bianchi, "Performance analysis of the IEEE 802.11 distributed coordination function," IEEE Journal on Selected Areas in Communications, vol. 18, no. 3, pp. 535-547, 2000. 


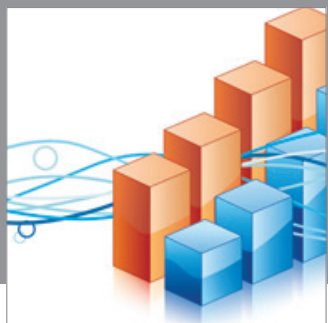

Advances in

Operations Research

mansans

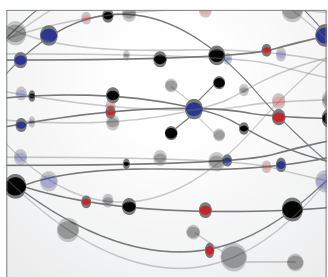

The Scientific World Journal
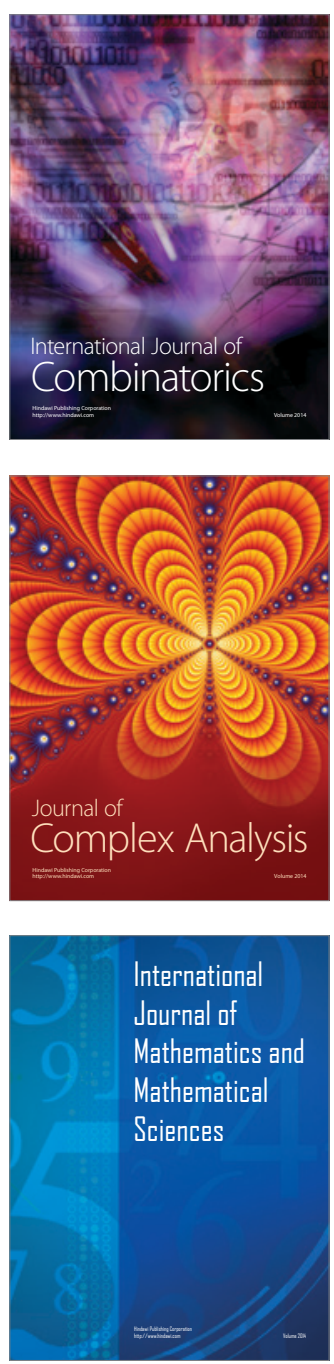
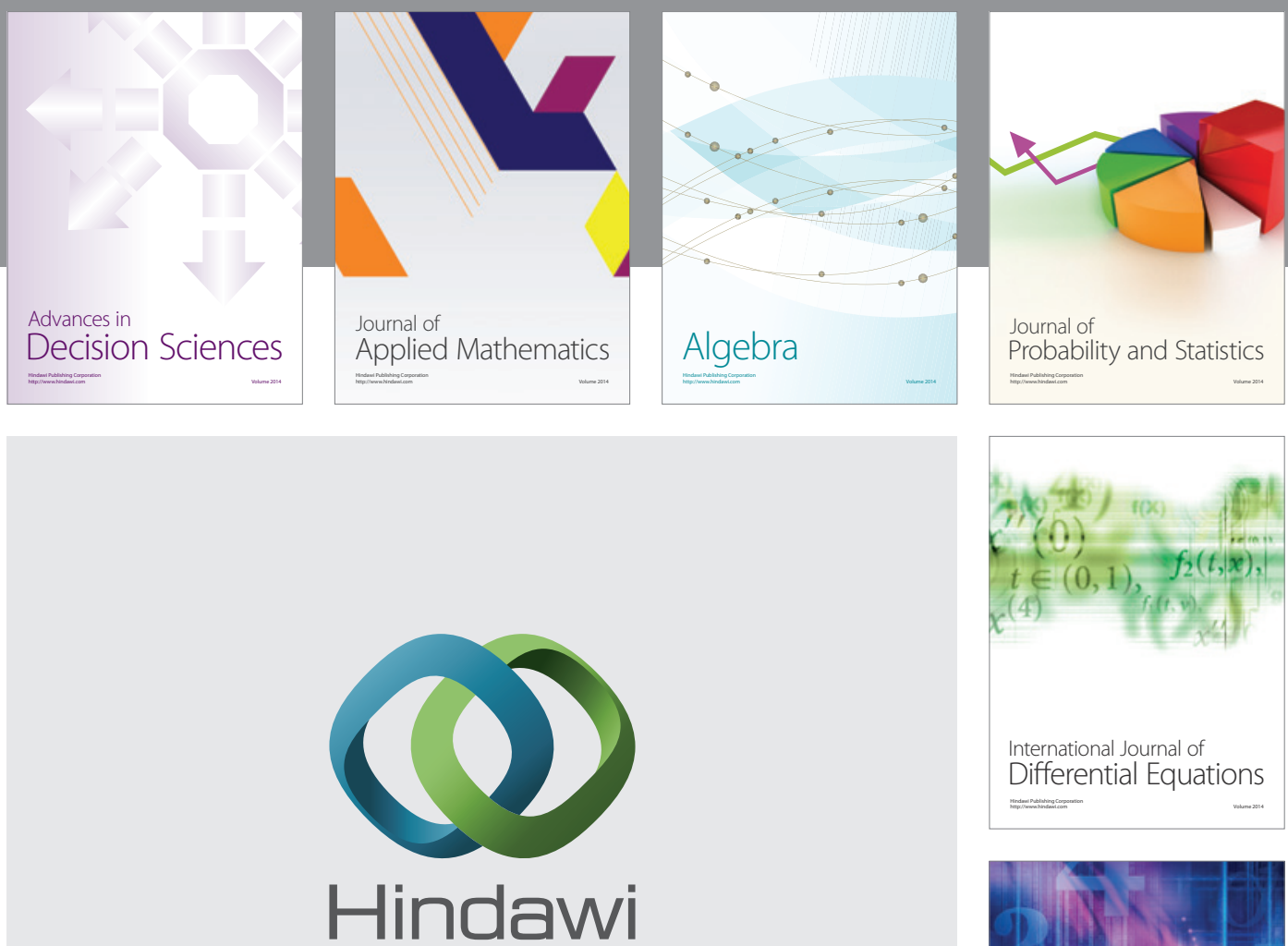

Submit your manuscripts at http://www.hindawi.com
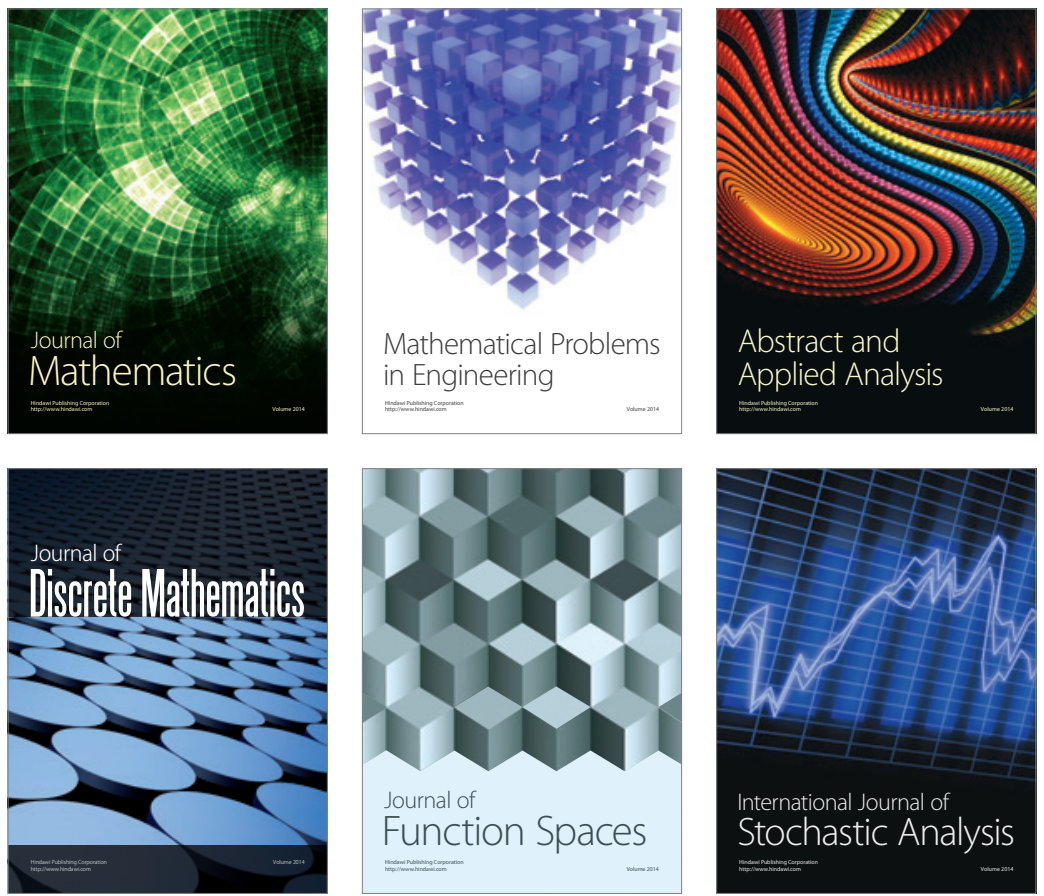

Journal of

Function Spaces

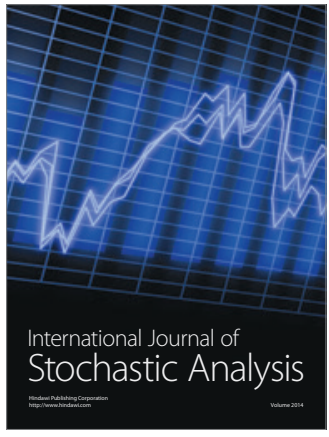

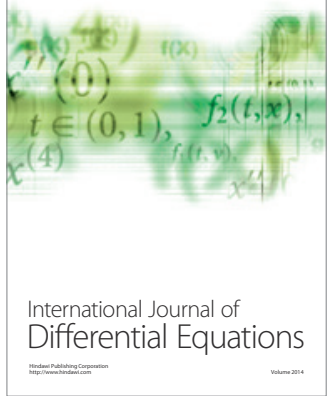
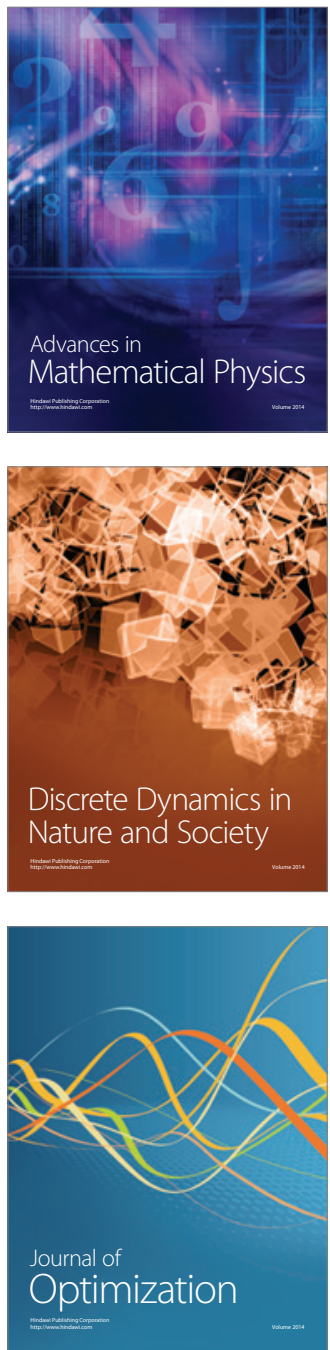